Supporting information

\title{
2D Perovskite Nanosheets with Intrinsic Chirality
}

\author{
He Ren ${ }^{1,2}$, Yue $\mathrm{Wu}^{1}$, Chenchen Wang ${ }^{1,2}$, Yong Yan ${ }^{1,2,3 *}$ \\ ${ }^{1}$ CAS Key Laboratory of Nanosystem and Hierarchical Fabrication, CAS Center for \\ Excellence in Nanoscience, National Center for Nanoscience and Technology, Beijing \\ 100190, China \\ ${ }^{2}$ University of Chinese Academy of Sciences, Beijing, 100049, China \\ ${ }^{3}$ Department of Chemistry, School of Chemistry and Biological Engineering, University \\ of Science and Technology Beijing, Beijing 100083, China
}

*Corresponding author. E-mail: yany@nanoctr.cn 


\section{Experimental section}

\section{Chemicals}

$R$ - $\beta$-methylphenethylamine ( $R$-MPEA) $(99 \%)$ and $S$ - $\beta$-methylphenethylamine $(S$ MPEA) (99\%) were purchased from Heowns Biochem Technologies Co., Ltd. $R$ 2-butylamine ( $R$-BA) and $R$-2-hexylamine $(R$-HA) were obtained from Alfa Aesar. $\mathrm{PbBr}_{2}(99.9 \%)$ was purchased from Macklin Biochemical Co., Ltd. $R$ - $\alpha-$ methylbenzylamine ( $R$-MBA), hydrobromide acid (HBr, 48 wt.\%), octylamine (99.5\%), oleic acid (90\%), and $N, N$-dimethylformamide (DMF, 99.8\%) were obtained from Aladdin. Diethyl ether, toluene, and isopropyl were used as received without further purification.

\section{Synthesis of precursor}

The precursors $R$ - $\beta$-methylphenethylammonium bromide ( $R$-MPEABr) and $S$ - $\beta$ methylphenethylammonium bromide (S-MPEABr) were prepared according to previous report. ${ }^{23}$ In a typical synthesis, $0.79 \mathrm{~mL}$ of $\mathrm{HBr}(48 \mathrm{wt} . \%)$ aqueous solution was added (dropwise) into a mixture of $10 \mathrm{~mL}$ isopropanol and $1 \mathrm{~mL} R$ - or $S$-MPEA under stirring. The reaction mixture was always kept in an ice bath. After 3 hrs, the solvent was removed by using a rotary evaporator to yield the $R$ - or $S$-MPEABr as a white powder. The product was purified by performing dissovling/precipitating cycles twice using diethyl ether and isopropanol, respectively. Finally, the pure white $R$ - or $S$-MPEABr was collected by drying at $60{ }^{\circ} \mathrm{C}$ for $24 \mathrm{hrs}$ under vacuum. To synthesize $R$ - $\mathrm{BABr}, R-\mathrm{HABr}$, and $R-\mathrm{MBABr}$, the same procedure was performed except for the use of different amines ( $R$-BA, $R$-HA, and $R$-MBA). 


\section{Synthesis of chiral perovskite nanocrystals}

In a typical synthesis, the growth solution was firstly prepared by dissolving chiral ammonium bromide (e.g. R-MPEABr, $0.0216 \mathrm{~g}), \mathrm{PbBr}_{2}(0.0147 \mathrm{~g})$, and $100 \mu \mathrm{L}$ oleic acid in $1 \mathrm{~mL}$ DMF. $20 \mu \mathrm{L}$ growth solution was then quickly injected into $5 \mathrm{~mL}$ of toluene under vigorous stirring at room temperature. The reaction proceed for $2 \mathrm{~min}$ and the unreacted materials and aggregated precipitate were removed by centrifuging at $3500 \mathrm{rpm}$ for $3 \mathrm{~min}$. Finally, the chiral perovskite nanocrystals were collected by centrifuging at $9000 \mathrm{rpm}$ for $3 \mathrm{~min}$ and redispersed in toluene.

\section{Characterization}

Transmission electron microscopy (TEM) images were collected by using FEI Tecnai G2 F20 U-TWIN microscope with an acceleration voltage of $200 \mathrm{kV}$. AFM images were captured by using a Bruker multimode 8 microscope in the tapping mode. The X-ray diffraction (XRD) patterns were recorded by using Rigaku D/MAX-TTRIII 3 (CBO) X-ray diffractometer with $\mathrm{Cu} \mathrm{K} \alpha(\lambda=1.5406 \AA)$ radiation source. The UV-vis, photoluminescence, and circular dichroism spectra were measured by using Shimadzu UV-2550 spectrometer, Horiba jobin-Yvon Fluorolog-III spectrofluoroemeter, and Jasco J-810 CD spectrometer, respectively. 
(a)<smiles>CC[C](C)N</smiles><smiles>CCCC[C@H](C)N</smiles>

R-HA

(b)

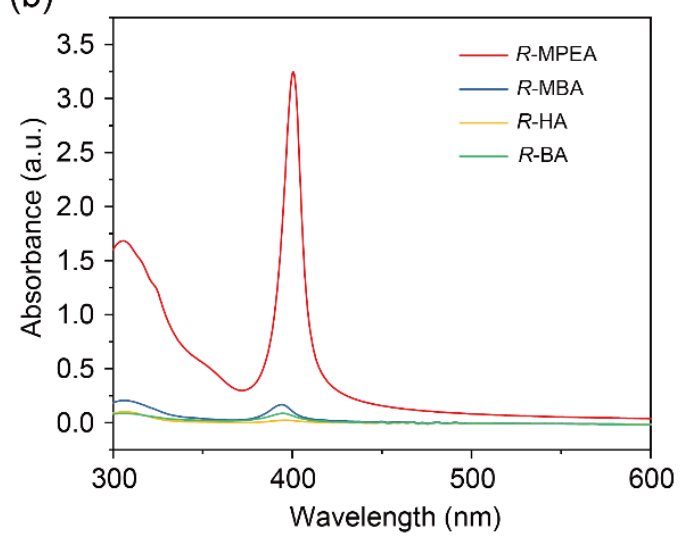<smiles>CC(N)c1ccccc1</smiles>

R-MBA

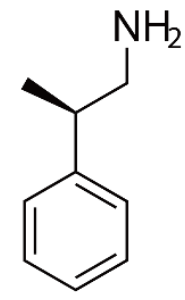

R-MPEA

(c)

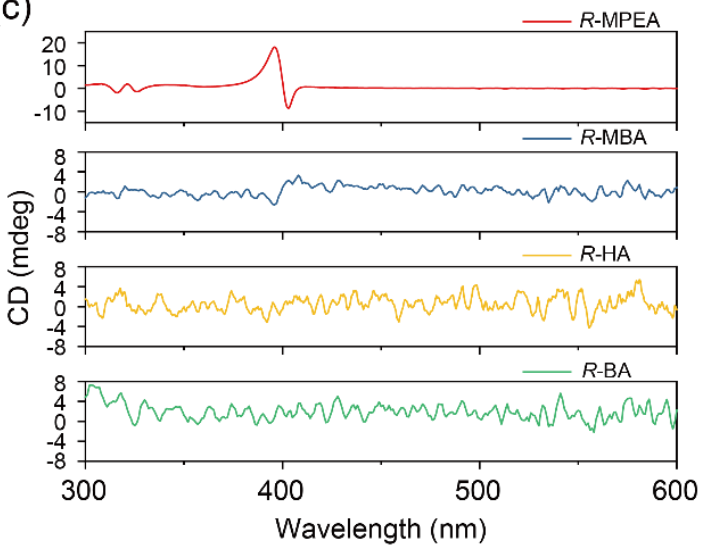

Fig. S1 Perovskite NCs prepared by using four types of amines with different reactivity.

(a) Chemical structure of $R$-2-butylamine, $R$-2-hexylamine, $R$ - $\alpha$-methylbenzylamine, and $R$ - $\beta$-methylphenethylamine. Absorption (b) and CD (c) spectra of four type of perovskite NCs prepared by using chiral amines in (a). 

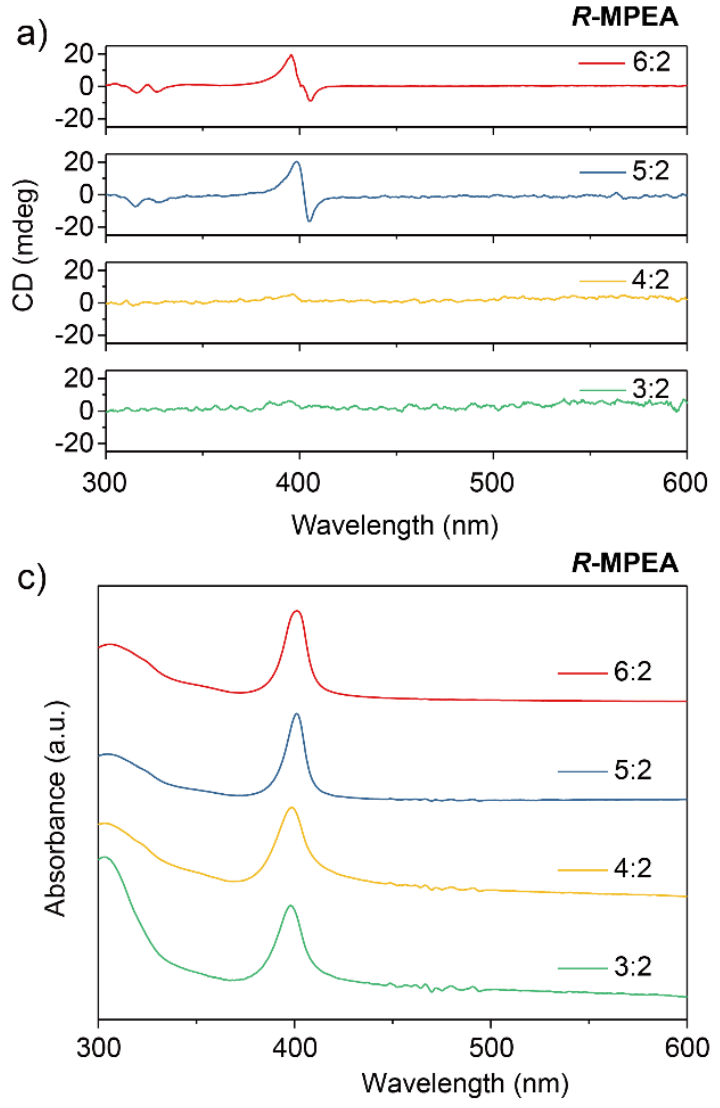
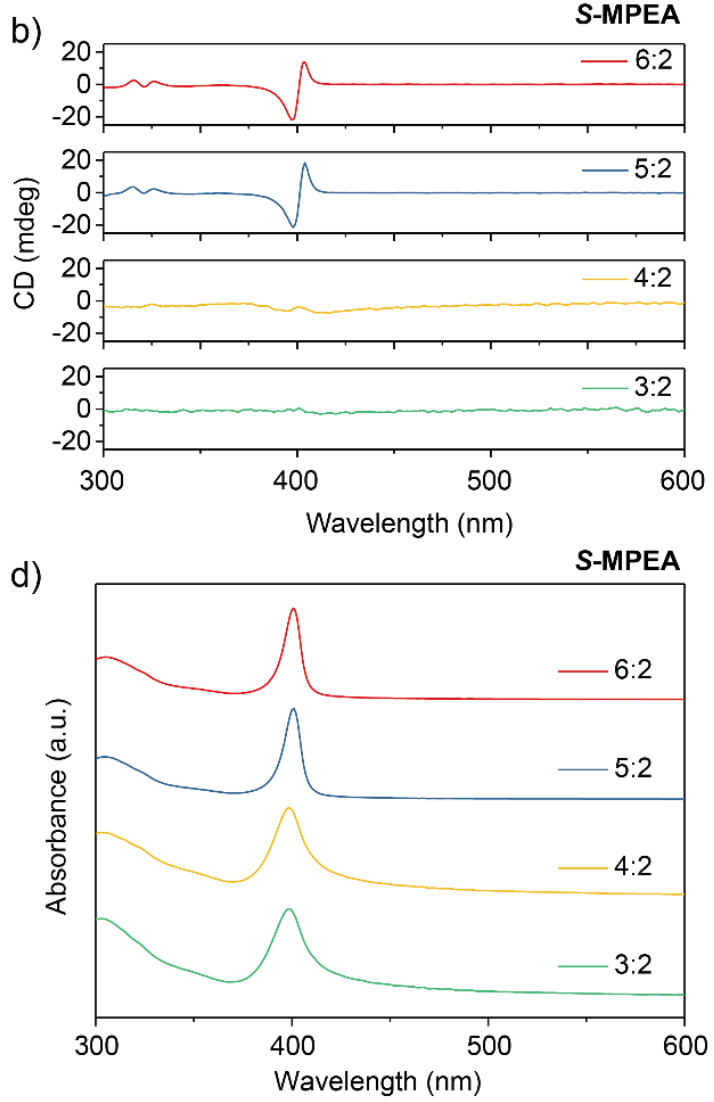

Fig. S2 CD spectra of chiral perovskite NCs prepared with different ratios of (a) $R$ MPEABr, (b) $S$-MPEABr and $\mathrm{PbBr}_{2}$. (c) and (d) Corresponding absorption spectra of perovskite NCs with $R$ - and $S$-MPEA, respectively. 

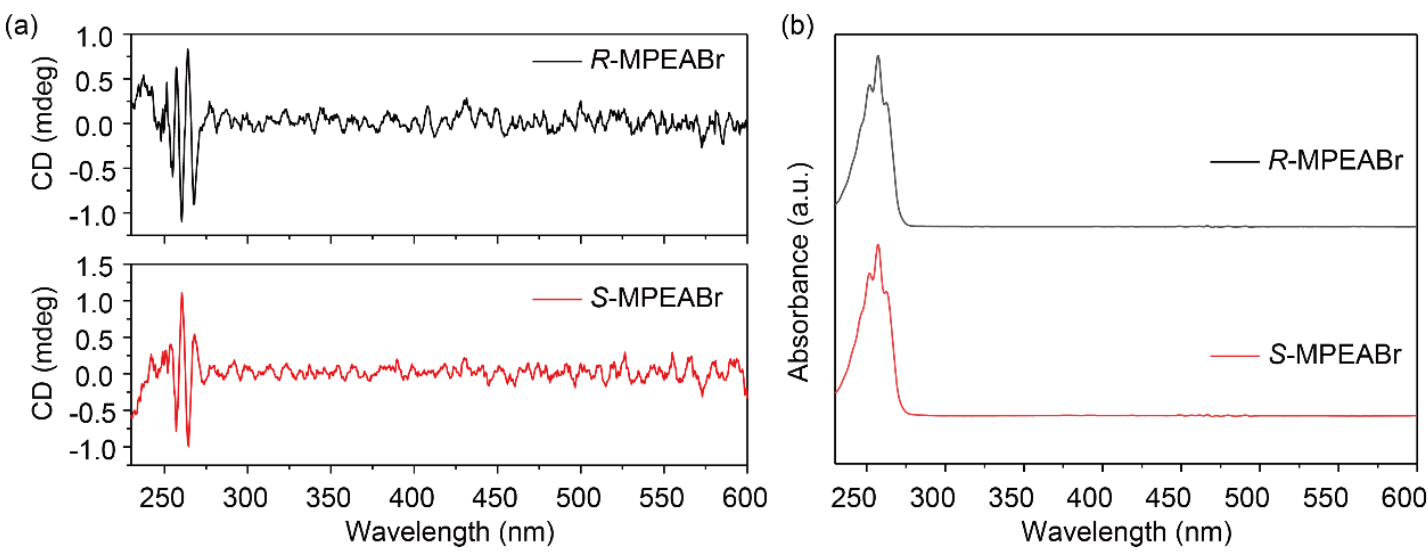

Fig. S3 (a) CD and (b) absorption spectra of $R$-MPEABr (black) and S-MPEABr (red) measured in ethanol.
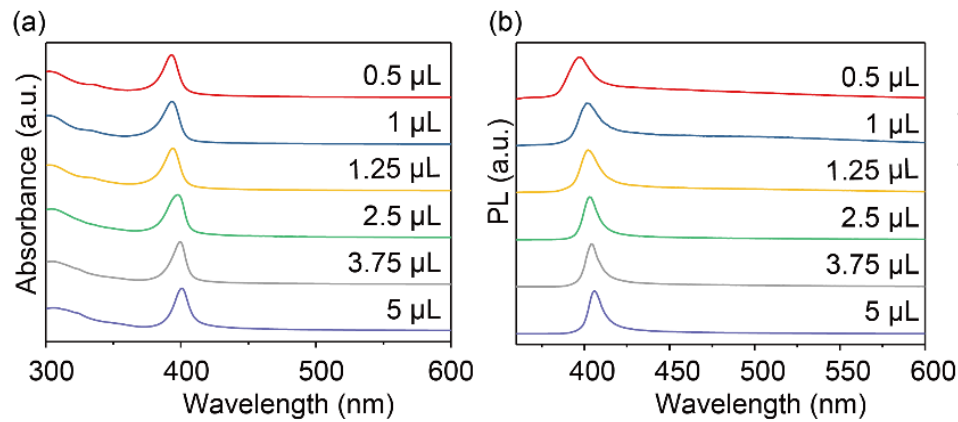

(c)

Fig. S4 (a) Absorption, (b) PL, and (c) XRD spectra of S-MPEA perovskite NSs prepared by using different amount of OA.

(a)

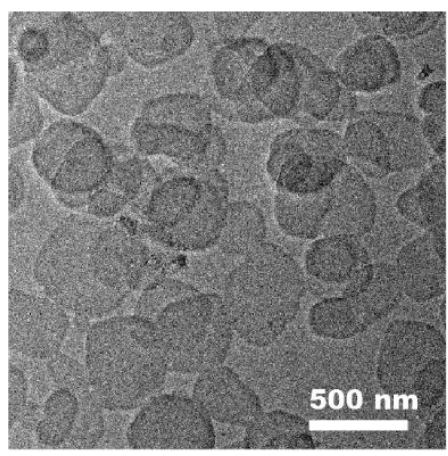

(b)

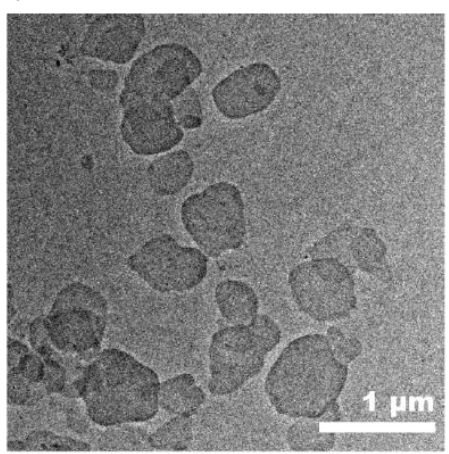

(c)

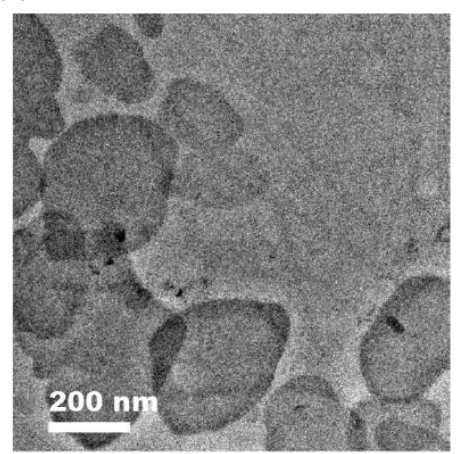

Fig. S5 TEM images of $R$-MPEA perovskite NSs prepared by using (a) $5 \mu \mathrm{L}$, (b) 3.75 $\mu \mathrm{L}$, and (c) $2.5 \mu \mathrm{L}$ OA. 